\title{
Influence of dysprosium addition on the structural, morphological, electrical and magnetic properties of nano-crystalline $\mathrm{W}$-type hexaferrites
}

\author{
ALI-SHARBATI*, JAVAD-MOLA VERDI KHANI, G R AMIRI and R MOUSAREZAEI \\ Falavarjan Branch, Islamic Azad University, Isfahan 8315734317, Iran
}

MS received 6 November 2013; revised 30 March 2014

\begin{abstract}
Dysporium (Dy)-substituted W-type barium hexaferrites were prepared by the citrate sol-gel-method. Crystalline structure, morphology, magnetic properties, DC resistivity and microwave absorption properties of $\mathrm{BaNi}_{2} \mathrm{Dy}_{x} \mathrm{Fe}_{16-x} \mathrm{O}_{27}(x=0-0.9)$ were studied using X-ray diffraction, transmission electron microscope (TEM), vibrating sample magnetometer and vector network analyzer and sensitive source meter, respectively. Single-phase W-type barium hexaferrites, with a chemical composition of $\mathrm{BaNi}_{2} \mathrm{Dy}_{x} \mathrm{Fe}_{16-x} \mathrm{O}_{27}(x=0-0.9)$, were formed by being heated at $1250^{\circ} \mathrm{C}$ for $4 \mathrm{~h}$ in air. The magnetic properties such as saturation magnetization $\left(M_{\mathrm{s}}\right)$, and coercivity $\left(H_{\mathrm{c}}\right)$ were calculated from hysteresis loops. Hysteresis loop measurements show that the coercivity values lie in the range of 530-560 Oe with increasing Dy content. It was also observed that magnetization decreases with the increase of Dy content. The DC resistivity was observed to increase from $0.83 \times 10^{7}$ to $6.92 \times 10^{7} \Omega \mathrm{cm}$ with increasing Dy contents due to the unavailability of $\mathrm{Fe}^{3+}$ ions. Microwave absorption properties of hexaferrite $(70 \mathrm{wt} \%)$-acrylic resin (30 wt \%) composites were measured by the standing-wave-ratio (SWR) method in the range from 12 to 20 GHz. For sample with $x=0.6$, a minimum reflection loss of $-40 \mathrm{~dB}$ was obtained at $16.2 \mathrm{GHz}$ for a layer of $1.7 \mathrm{~mm}$ in thickness. Sample with $x=0.9$ had wide bandwidth absorption in the frequency range of 13.5-18 GHz with reflection losses less than $\mathbf{- 1 5} \mathrm{dB}$. Meanwhile the minimum reflection point shifts toward higher frequency with the increase of Dy content.
\end{abstract}

Keywords. Nano-crystalline materials; magnetic materials; magnetic properties; chemical synthesis.

\section{Introduction}

Hexaferrites are classified into six types depending on chemical formulae and crystal structure. These include M-type, W-type, X-type, Y-type, U-type and Z-type. They have been used in a variety of applications and attracted much attention over the years. M-type hexaferrite is the most popular hard magnetic material in the industry; W-type hexagonal ferrite is a type of crucial gyromagnetic materials and Z-type and Y-type hexagonal ferrites are excellent soft magnetic materials in very high frequency (VHF) and ultra-high frequency (UHF). ${ }^{1-6}$ In the recent years, a number of researchers have attempted the substitution of divalent ions, trivalent ions and their binary mixture in order to alter the structural, electrical and magnetic properties of W-type hexaferrites. ${ }^{7,8}$ The crystal structure of W-type hexagonal ferrites is very complex and can be considered as a superposition of $\mathrm{R}$ and $\mathrm{S}$ blocks along the hexagonal axis with a structure of $\mathrm{RSSR} * \mathrm{~S} * \mathrm{~S} *$, where $\mathrm{R}$ is a three-oxygen-layer block with composition $\mathrm{BaFe}_{6} \mathrm{O}_{11}, \mathrm{~S}$ (spinel block) is a two-oxygen-layer block with composition $\mathrm{Fe}_{6} \mathrm{O}_{8}$ and the asterisk means that the corresponding block has been turned $180^{\circ}$ around the hexagonal axis. ${ }^{9}$ W-type

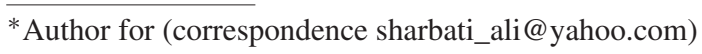

hexaferrites continues to be very attractive materials for home appliances, electronic products, communication equipments and data processing devices because of their unique electrical and magnetic properties. ${ }^{10}$ The magnetic and dielectric behaviour in W-type hexaferrite depends on many factors, such as preparation method, calcining temperature, amount and type of substitution, etc. ${ }^{11}$ Rare-earth (RE) ions take on unpaired $4 f$ electrons and the strong spin-orbit coupling of their angular momentum improves the overall electrical and magnetic properties. Using RE as dopants has been reported as an effective method in varying magnetic properties of ferrite materials. Many reports have recently shown that REsubstituted W-hexaferrites have exhibited improved magnetic properties. ${ }^{12,13} \mathrm{RE}$ elements have typical magnetocrystalline anisotropy and high coercivity, which may affect the electromagnetic properties of ferrite magically. ${ }^{14,15}$ Usually the $\mathrm{RE}$ ions were substituted for $\mathrm{Sr}(\mathrm{Ba})$ or $\mathrm{Fe}$, taking into accounts the ionic radii of the elements. ${ }^{16} \mathrm{RE}$ elements can also be used as inhibitor agents of the grain growth mechanism at high temperature. ${ }^{17}$ According to this approach, we synthesized dysporium (Dy)-substituted W-type hexagonal ferrites and to study their structural, magnetic, microwave absorption and electrical properties of $\mathrm{BaNi}_{2} \mathrm{Dy}_{x} \mathrm{Fe}_{16-x} \mathrm{O}_{27}$ powders. We report here an investigation of the morphological, structural, magnetic and surface 
compositional characteristics of the products which has been carried out by means of X-ray powder diffraction (XRD), transmission electron microscope (TEM), vibrating sample magnetometer (VSM), vector network analyzer and electrical resistivity.

\section{Experimental}

A series of samples was prepared by the citrate sol-gel method with composition $\mathrm{BaNi}_{2} \mathrm{Dy}_{x} \mathrm{Fe}_{16-x} \mathrm{O}_{27}$ where in $x=$ $0.0,0.3,0.6$ and 0.9. First, stoichiometric amounts of ferric nitrate, barium nitrate, dysprosium oxide, and nickel nitrate were dissolved together in a minimum amount of citric acid aqueous solution at $80^{\circ} \mathrm{C}$ according to the formula of $\mathrm{BaNi}_{2} \mathrm{Dy}_{x} \mathrm{Fe}_{16-x} \mathrm{O}_{27}(x=0.0,0.3,0.6$ and 0.9$)$. The molar ratios of citric acid to metal ions used were $1: 1$. Then, ammonia solution was added drop by drop to increase the $\mathrm{pH}$ to facilitate the formation of a homogeneous solution, and the solution was slowly evaporated until a highly viscous residue was formed. $\mathrm{pH}$ of the solution was adjusted to 7.0. After that, the residue was dried immediately in an oven in the temperature range of $120-140^{\circ} \mathrm{C}$. Finally, the dry gel was calcined at $1250^{\circ} \mathrm{C}$ for $4 \mathrm{~h}$.

XRD analysis of powder samples were carried out by $\mathrm{X}$-ray diffractometer equipped with $\mathrm{CuK}_{\alpha}$ radiation source $(\lambda=1.5406 \AA)$. The magnetic properties like magnetization values $\left(M_{\mathrm{s}}\right)$ and coercivity $\left(H_{\mathrm{c}}\right)$ were measured by a VSM at an applied field of $12 \mathrm{kOe}$ at room temperature. The particle morphology was examined by TEM JEOL1230 with an accelerating voltage of $200 \mathrm{kV}$. The electrical properties were measured by two-probe method, using sensitive source meter model Keithly 2400.

The composite specimens were prepared by mixing doped strontium ferrite and acrylic resin powders with concentrations of 70:30 by weight. Mixture of ferrite powders with acrylic resin powders were plasticized and heated at $220^{\circ} \mathrm{C}$ and 5.5 $\mathrm{MPa}$. The pressed composites were fabricated in cylindrical form with a thickness of $1.7 \mathrm{~mm}$ and a constant diameter of $40 \mathrm{~mm}$. The reflection loss of the absorbers was calculated from the standing-wave-ratio (SWR) measured as a function of frequency using vector network analyzer (R\&SZVK). ${ }^{16}$ The vector network analyzer was fully calibrated before use.

\section{Results and discussion}

\subsection{Crystal structure}

The XRD patterns of the synthesized hexaferrite materials are presented in figure 1. XRD analysis reveals that all the observed diffraction peaks match well with the standard pattern of W-type hexaferrite (ICDD 00-051-1879). The observed peaks perfectly match with the standard pattern and have no extra peak except that of the pure W-type hexaferrites. All the samples show good crystallization, with well-defined diffraction lines. It is observed from figure 1

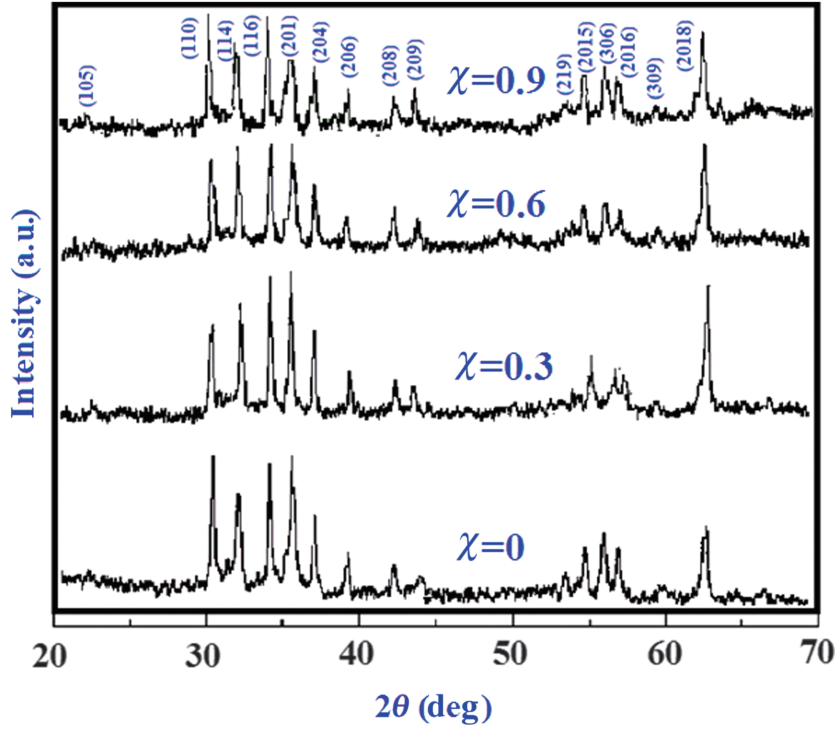

Figure 1. XRD pattern of calcined powders at $1200^{\circ} \mathrm{C}$ : (a) $x=0$, (b) $x=0.3$, (c) $x=0.6$ and (d) $x=0.9$.

Table 1. The structural and magnetic parameters of $\mathrm{BaNi}_{2} \mathrm{Dy}_{x}$ $\mathrm{Fe}_{16-x} \mathrm{O}_{27}(x=0-0.9)$.

\begin{tabular}{lccccccc}
\hline & $\begin{array}{c}c \\
(\mathrm{~nm})\end{array}$ & $\begin{array}{c}c \\
(\mathrm{~nm})\end{array}$ & $c / a$ & $\begin{array}{c}V \\
(\mathrm{~nm})^{3}\end{array}$ & $\begin{array}{c}D \\
(\mathrm{~nm})\end{array}$ & $\begin{array}{c}H_{\mathrm{c}} \\
(\mathrm{Oe})\end{array}$ & $\begin{array}{c}M_{\mathrm{s}} \\
\left(\mathrm{emu} \mathrm{g}^{-1}\right)\end{array}$ \\
\hline 0 & 0.5866 & 3.3261 & 5.670 & 0.991 & 33 & 530 & 74 \\
0.3 & 0.5870 & 3.3266 & 5.667 & 0.992 & 35 & 542 & 70 \\
0.6 & 0.5873 & 3.3270 & 5.664 & 0.993 & 39 & 548 & 60 \\
0.9 & 0.5877 & 3.3273 & 5.661 & 0.995 & 45 & 560 & 53 \\
\hline
\end{tabular}

that the increase in the $\mathrm{Dy}^{3+}$ content causes the change in the intensity of the XRD characteristic peaks of $\mathrm{BaNi}_{2} \mathrm{Fe}_{16} \mathrm{O}_{27}$. Lattice parameters ' $a$ ' and ' $c$ ', as shown in table 1, are calculated from the XRD data using equation

$$
\frac{1}{d_{h k l}^{2}}=\frac{4}{3}\left(\frac{h^{2}+k^{2}+l^{2}}{a^{2}}\right)+\frac{l^{2}}{c^{2}},
$$

where $h, k$ and $l$ are the Miller indices and $d$ the interplaner spacing. The value for the lattice parameters ' $a$ ' and ' $c$ ' increases regularly with the addition of Dy ion whereas there is a little or no variation in the $d$-spacing values with respect to the 2 -theta values. The observed phenomenon can be justified on the basis that the ionic radii of dysprosium $(0.104 \mathrm{~nm})$ is comparatively higher than the ionic radius of $\mathrm{Fe}^{3+}(0.64 \AA)$. The average crystallite size of the samples is determined from the 'full-width at half-maxima' (FWHM) of (201) reflection by using the Debye-Scherrer formula $^{18}$

$$
D=\frac{0.9 \lambda}{\beta \cos \theta_{\mathrm{B}}},
$$


where $k$ is the shape constant, $\beta$ the broadening of diffraction line measured at half-width of maximum intensity and $\lambda$ the X-ray wavelength used for the analysis, i.e., (1.542 $\AA$ ). The calculated crystallite size of all the samples is in the range of 33-45 nm.

Figure 2 shows the TEM micrograph of synthesized particles by the sol-gel combustion. The TEM micrograph of nanoparticles indicates that the particles have a distribution of particle size from 40 to $50 \mathrm{~nm}$. The images show that ferrite particles possess irregular non-spherical shapes with a broad size distribution. The images also clearly indicate that the particles are stacked on top of each other because of their mutual magnetic interaction.

\subsection{Electrical and magnetic properties}

The $M-H$ loops of all samples ( $x=0,0.3,0.6$ and 0.9$)$ are shown in figure 3 . The values of coercive force $\left(H_{\mathrm{c}}\right)$ and saturation magnetization $\left(M_{\mathrm{s}}\right)$ were calculated for each composition and are listed in table 1. Results show that the coercive force $\left(H_{\mathrm{c}}\right)$ increases with the increase of Dy
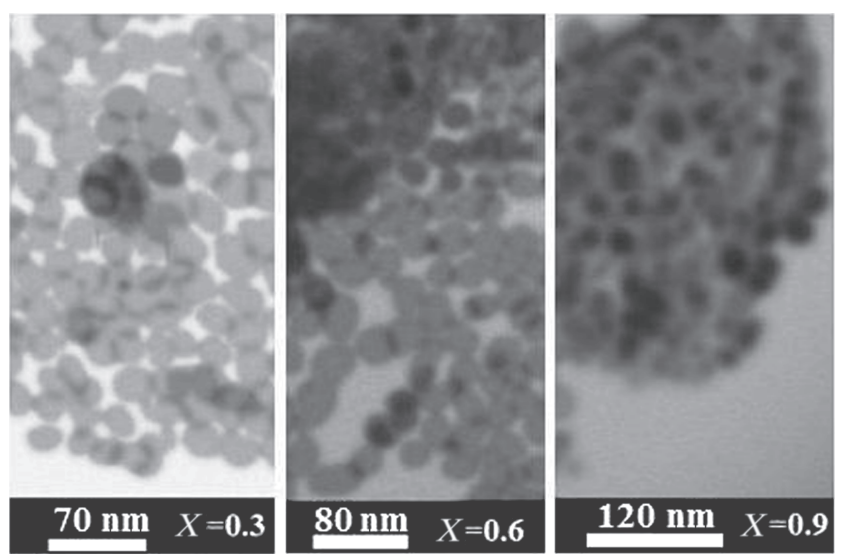

Figure 2. TEM micrographs of prepared powders.

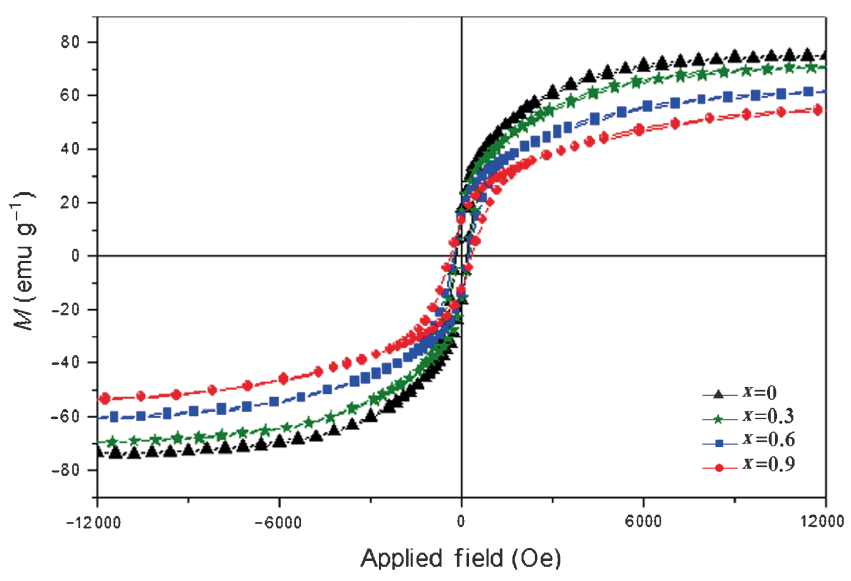

Figure 3. Room temperature hysteresis loops of (a) $x=0$, (b) $x=0.3$, (c) $x=0.6$ and (d) $x=0.9$. concentration. The increase in $H_{\mathrm{c}}$ for samples can be attributed to an enhancement of the magnetocrystalline anisotropy ${ }^{17}$ with anisotropic $\mathrm{Fe}^{2+}$ ions locating on a $2 a$ site and the grain size reduction which is usually found with RE substitutions, which is evident in table $1 .{ }^{19,20} \mathrm{It}$ should be noted that it is a well-known fact that the coercive force is heavily dependent upon grain size. ${ }^{21}$ Also, our results show that saturation magnetization decreases with the increase in the Dy concentration. This behaviour may be due to the difference between the ionic radii of $\mathrm{Dy}^{3+}$ and $\mathrm{Fe}^{3+}$ which introduces local strain that may cause the disorder and modifications of local electronic states. ${ }^{20}$ In general the RE substitutions weaken the super exchange interactions between Fe sites because of its strongly distorted environment ${ }^{22}$ resulting in the reduction of total magnetic moment.

The room temperature resistivity of all samples $(x=0$, 0.3, 0.6 and 0.9) are shown in figure 4. As shown in figure 4, the room temperature resistivity increases from a value $0.83 \times 10^{7}$ to $6.92 \times 10^{7} \Omega \mathrm{cm}$ with increasing Dy concentration. Hence the present samples can be used at higher frequencies due to low eddy current losses. ${ }^{5}$ As Dy ions prefer to occupy octahedral sites followed by the migration of some $\mathrm{Fe}^{3+}$ ions to tetrahedral sites and converting them into $\mathrm{Fe}^{2+}$ ions in order to maintain overall electrical neutrality. As a result $\mathrm{Fe}^{3+}$ ions concentration is lowered on octahedral sites: the sites responsible for conduction in ferrites. All these factors would limit the hopping probability between $\mathrm{Fe}^{3+}$ and $\mathrm{Fe}^{2+}$ ions thereby enhancing the resistivity.

\subsection{Attenuation properties}

Electromagnetic (EM) wave attenuation is determined from the reflection loss (RL). In the case of a metal-backed single layer, RL is given Sharbati and Mola Verdi Khani ${ }^{16}$

$$
\begin{aligned}
\mathrm{RL}(\mathrm{dB}) & =20 \log \left|\frac{Z_{\text {in }}-1}{Z_{\text {in }}+1}\right|, \\
Z_{\text {in }} & =\sqrt{\frac{\mu_{\mathrm{r}}}{\varepsilon_{\mathrm{r}}}} \tanh \left[j \frac{2 \pi}{c} \sqrt{\mu_{\mathrm{r}} \varepsilon_{\mathrm{r}}} f d\right],
\end{aligned}
$$

where $Z_{\text {in }}$ is the impedance of the composites, $Z_{0}$ the intrinsic impedance of free space, $c$ the light velocity, $d$ the thickness of composite, $f$ the frequency of the incident EM wave, $\mu$ the complex permeability and $\varepsilon$ the complex permittivity. The impedance matching condition is given by $Z_{\text {in }}=1$, to represent the perfect absorbing properties. The $10 \mathrm{~dB}$ absorbing bandwidth means that the frequency bandwidth can achieve $90 \%$ of reflection loss, whereas a $20 \mathrm{~dB}$ absorbing bandwidth means that the frequency bandwidth can achieve $99 \%$ of reflection loss.

In general, when polycrystalline ferrites are placed in an ac field, two different resonance mechanisms are produced, i.e., the natural resonance and the domain wall resonance. For spinel ferrites, many experiments and analyses have 


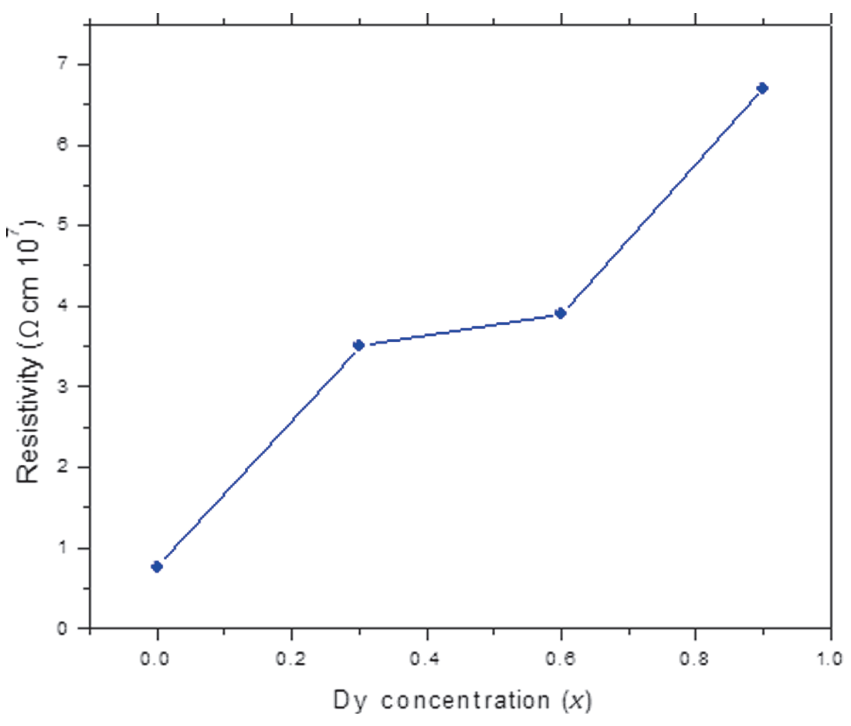

Figure 4. Electrical resistivity vs. Dy concentration $(x)$.

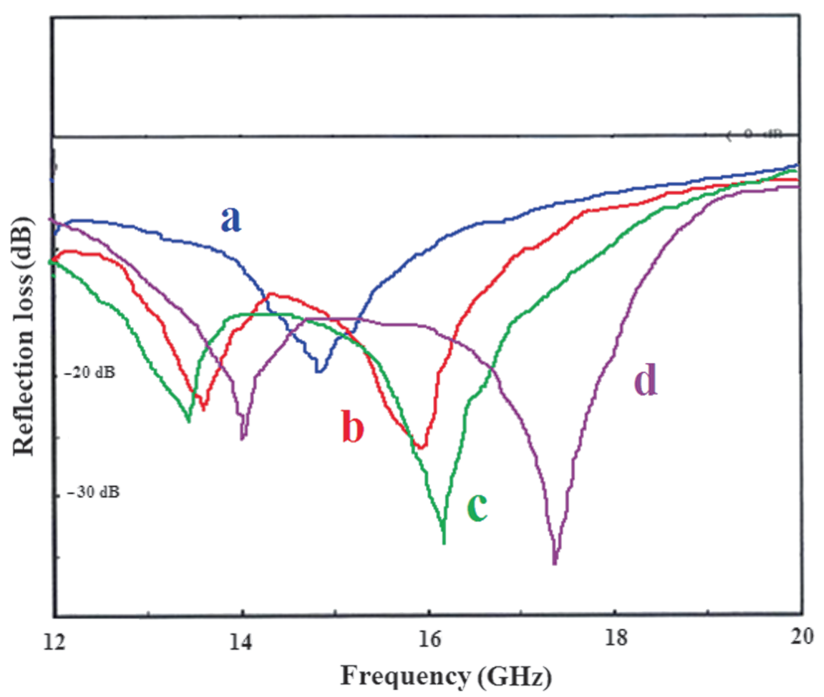

Figure 5. Absorption characteristics of the composite: (a) $x=0$, (b) $x=0.3$, (c) $x=0.6$ and (d) $x=0.9$.

shown that the natural resonance frequency is higher than the domain wall resonance.

The following relation gives the ferromagnetic resonance frequency for the barium hexaferrite

$$
f_{\mathrm{R}}=\frac{1}{2 \pi} \eta\left(H_{\theta} H_{\varphi}\right)^{1 / 2},
$$

where $\eta$ is the gyromagnetic ratio and $H_{\theta}$ and $H_{\varphi}$ the out-of-plane and in-plane anisotropy fields, respectively. Equation (2) shows that the ferromagnetic resonance frequency depends on the magnetocrystalline anisotropy fields of barium ferrites, $H_{\theta}$ and $H_{\varphi}$.

Figure 5 shows the variation of reflection loss as a function of frequency for the hot-pressed hexaferrite composites with $x=0.0,0.3,0.6$ and 0.9 and of $1.7 \mathrm{~mm}$ thickness. As shown in figure 5 , sample ' $x=0$ ' shows a minimum reflection loss of $-19 \mathrm{~dB}$ at $14.9 \mathrm{GHz}$, sample ' $x=0.3$ ' shows $-26 \mathrm{~dB}$ at $16.1 \mathrm{GHz}$, sample ' $x=0.6$ ' shows $-40 \mathrm{~dB}$ at $16.2 \mathrm{GHz}$ and sample ' $x=0.9$ ' shows $-42 \mathrm{~dB}$ at $17.4 \mathrm{GHz}$. The frequency range for attenuation higher than $10 \mathrm{~dB}$ covers $13.5-18 \mathrm{GHz}$ for sample ' $x=0.9$ ', $12.9-17.2 \mathrm{GHz}$ for sample ' $x=0.6$ ', $13.1-14$ and $15-16.2 \mathrm{GHz}$ for sample ' $x=0.3$ ' and $14.1-15.3 \mathrm{GHz}$ sample ' $x=0$ '. Also, results indicate that the peak in the absorption curves shift toward higher frequency by substitution of $\mathrm{Dy}^{3+}$ in the W-type hexaferrite samples.

\section{Conclusion}

Structural, magnetic properties and DC electrical resistivity properties of Dy-substituted Ni-W-type barium hexagonal ferrite for different compositions $(x=0.0-0.9)$ prepared via the citrate sol-gel method have been investigated. The ferrite with different Dy substitutions all composed of single W-type phase and the lattice parameters $c$ increases with increasing Dy substitution and the Dy substitutions have an effect on the cation occupation in the ferrites. The room temperature DC electrical resistivity increases by increasing Dy contents. Results show coercivity $\left(H_{\mathrm{c}}\right)$ increases from 530 to 560 Oe and saturation magnetization $\left(M_{\mathrm{s}}\right)$ decreases from 74 to $53 \mathrm{emu} \mathrm{g}^{-1}$ with increasing Dy substitution. The microwave absorbing properties were investigated for $\mathrm{BaNi}_{2} \mathrm{Dy}_{x} \mathrm{Fe}_{16-x} \mathrm{O}_{27}$ (70wt\%)-polymer (30\%) composites in the frequency range of $12-20 \mathrm{GHz}$. From these results, it can be concluded that the investigated ferrites may be expected to find potential application in reduction of EMI and RCS in stealth technology.

\section{References}

1. Albanese G, Deriu A, Licci F and Rinaldi S 1978 IEEE J. Trans. Magn. 14710

2. Deriu A, Licci S, Rinaldi F and Besagni T 1981J. Magn. Magn. Mater. 22257

3. Lee S G and Kwon S J 1996 J. Magn. Magn. Mater. 153279

4. Li Z W, Guoqing L, Chen L, Yuping W and Ong C K $2006 \mathrm{~J}$. Appl. Phys. 99063905

5. Iqbal M J and Khan R A 2009 J. Alloys Compd. 478847

6. Zhang H, Li L, Zhou J, Yue Z, Ma Z and Gui Z 2001 J. Eur. Ceram. Soc. 21149

7. Deng L, Ding L, Zhou K, Huang S, Hu Z and Yang B $2011 J$. Magn. Magn. Mater. 3231895

8. Guo F, Ji G, Xu J, Zou H, Gan S and Xu X 2012 J. Magn. Magn. Mater. 3241209

9. Hemeda D M, Al-Sharif A and Hemeda O M 2007 J. Magn. Magn. Mater. 315 L1

10. Ahmad M A, Okasha N and Kershi R M 2009 J. Magn. Magn. Mater. 3213967 
11. Zhang H, Liu Z, Yao X, Zhang L and Wu M 2003 J. Sol-Gel Sci. Technol. 27277

12. Ahmad M A, Okasha N and Kershi R M 2010 Physica B $\mathbf{4 0 5}$ 3232

13. Xu J, Zou H, Li H, Li G, Gan S and Hong G 2010 J. Alloys Compd. 490552

14. Jing W, Hong Z, Shuxin B, Ke C and Changrui Z 2007 J. Magn. Magn. Mater. 312310

15. Meena R S, Bhattachrya S and Chatterjee R 2010 J. Magn. Magn. Mater. 3221923

16. Sharbati A and Mola Verdi Khani J 2013 J. Mater. Sci.: Mater. Electron. 243629
17. Ounnunkad S 2006 Solid State Commun. 138472

18. Iqbal M J, Khan R A, Mizukami S and Miyazaki T 2011 Mater. Res. Bull. 461980

19. Van Diepan A M and Lotgering F K 1974 J. Phys. Chem. Solids 351641

20. Sauer C, Kobler U, Zinn W and Stablein H 1978 J. Phys. Chem. Solids 391197

21. Sharbati A, Choopani S, Mousavi Azar A and Senna M 2012 Solid State Commmun. 1502218

22. Rezlescu N, Doroftei C, Rezlescu E and Popa P D $2008 \mathrm{~J}$. Alloys Compd. 451492 